that allows simultaneous detection of organisms associated with BV, VVC and TV, we sought to characterize BV marker combinations in the absence and presence of $\mathrm{BV}$ and/or $\mathrm{TV}$ co-infections.

Methods The BD MAX ${ }^{\text {тм }}$ Vaginal Panel is a sample to answer NAAT capable of simultaneous detection of $G$ vaginalis, $A$. vaginae, BVAB-2/Megaspheara-1, L. crispatus/L. jensenii, C. albicans/parapsilosis/tropicalis, C. glabrata, C. krusei and T. vaginalis. The data obtained from $\mathrm{BD}$ MAX Vaginal Panel runs conducted on 1,740 clinician-collected specimens taken from symptomatic patients was analyzed to determine if any detection patterns emerged. The distribution of BV marker combinations detected in the absence and presence of BV and/ or TV were compared.

Results Independent of the $\mathrm{BV}$ result $(\mathrm{BV}+$ or $\mathrm{BV}-)$, the proportions of samples containing no BV markers and samples containing all BV markers were different in $\mathrm{TV}-$ and $\mathrm{TV}+$ samples. TV $+/ \mathrm{BV}-$ samples displayed a significantly higher number of cases in which only A. vaginae was detected or a combination of $A$. vaginae and $G$. vaginalis than in samples found $\mathrm{TV}-/ \mathrm{BV}-$, $\mathrm{TV}-/ \mathrm{BV}+$ or $\mathrm{TV}+/ \mathrm{BV}+$.

Conclusion The BV marker detection patterns vary with the presence of co-infection by TV. The results obtained in this analysis suggest some interplay between BV and TV and warrants further investigation.

Disclosure No significant relationships.

\section{P790 DETERMINING THE ORIGINS OF REPEAT TRICHOMONAS VAGINALIS INFECTIONS USING CLINICAL VERSUS GENOTYPE-INFORMED CRITERIA}

\begin{abstract}
${ }^{1}$ Patricia Kissinger*, ${ }^{2}$ Martina Bradic, ${ }^{3}$ Christina Muzny, ${ }^{4}$ Leandro Mena, ${ }^{5}$ Rebecca Lillis, ${ }^{3}$ Jane Schwebke, ${ }^{4}$ Laura Beauchamps, ${ }^{5}$ Stephanie Taylor, ${ }^{6}$ Norine Schmidt, ${ }^{7}$ Peter Augostini, ${ }^{7}$ William Secor, ${ }^{2}$ Jane Carlton, ${ }^{6}$ David Martin. ${ }^{1}$ Tulane School of Public Health and Tropical Medicine, New Orleans, USA; ${ }^{2}$ New York University, Center for Genomics and Systems Biology, New York, USA; ${ }^{3}$ University of Alabama at Birmingham, Medicine, Birmingham, USA; ${ }^{4}$ University of Mississippi Medical Center, Medicine, Jackson, USA; ${ }^{5}$ Louisiana State University, Department of Health Sciences, New Orleans, USA; ${ }^{6}$ Tulane University School of Public Health and Tropical Medicine, Epidemiology, New Orleans, USA; ${ }^{7}$ Centers for Disease Control and Prevention, Division of Parasitic Diseases and Malaria, Atlanta, USA
\end{abstract}

\subsection{6/sextrans-2019-sti.845}

Background High rates of repeat $T$. vaginalis infections posttreatment have been reported. It is essential to understand the origin of these infections (i.e. treatment failure or reinfection) to determine the best secondary prevention measures. Selfreported sexual behavior and medication adherence can be subject to bias. The purpose of this study is to examine the origins of early repeat $T$. vaginalis infections in women using clinical versus genotype-informed criteria.

Methods Women with $T$. vaginalis confirmed by culture or nucleic acid amplification test (NAAT), who were randomized to receive $2 \mathrm{~g}$ or 7 -day $500 \mathrm{mg}$ BID metronidazole (MTZ), were retested 3-12 weeks post treatment at test-of-cure (TOC). Viable isolates from women who were TOC TV+ were genotyped (baseline and TOC isolates) and MTZ susceptibility (TOC only) was evaluated. Sexual and treatment adherence histories were elicited by computer-assisted selfadministered survey. Treatment failure was defined using two criteria: 1) clinical (a combination of MTZ adherent per selfreport + no follow-up sexual exposure per self-report + no MTZ resistance), and 2) genotype-informed (concordant baseline and TOC genotype with no follow-up sexual exposure per self-report).

Results Of 80 repeat positives, 78 were evaluated using clinical and 49 using genotype-informed criteria. Per clinical criteria, $87.2 \%$ were treatment failure, $7.7 \%$ were reinfection and $5.1 \%$ were new infection. Per genotype-informed criteria, $51.0 \%$ were treatment failure, $10.2 \%$ were reinfection and $38.3 \%$ were new infection. In subset analysis, comparing the 49 with both clinical and genotype-informed assessments, $61.2 \%$ agreed and 38.8\% disagreed (kappa 0.29 indicating poor reliability). Of 44 women who denied having a new partner during follow-up, 14 (31.8\%) had a discordant genotype.

Conclusion Using either criteria, most TOC T. vaginalis positives were treatment failure rather than re-infections. Clinical and genotype-informed classification were not well correlated Possible explanations for this will be discussed.

Disclosure No significant relationships.

\section{P791 ASSESSMENT OF URETHRITIS ETIOLOGY AMONG HIV- INFECTED MEN ATTENDING AN STI CLINIC IN LILONGWE, MALAWI}

${ }^{1}$ Mitch Matoga*, ${ }^{2}$ Jane Chen, ${ }^{3}$ Cecilia Massa, ${ }^{3}$ Beatrice Ndalama, ${ }^{4}$ Esther Mathiya ${ }^{4}$ Naomi Bonongwe, ${ }^{5}$ Blessings Kamtambe, ${ }^{3}$ Edward Jere, ${ }^{4}$ Edith Kamanga, ${ }^{6}$ Gerald Tegha, ${ }^{6}$ Tarsizio Chikaonda, ${ }^{7}$ Myron Cohen, ${ }^{7}$ Irving Hoffman. ${ }^{1}$ UNC Project Malawi, STI Research and Services, Lilongwe, Malawi; ${ }^{2}$ University of North Carolina, UNC Gillings School of Global Public Health, Chapel Hill, USA; ${ }^{3}$ UNC Project Malawi, Lilongwe, Malawi; ${ }^{4}$ UNC Project Lilongwe, STI Clinic, Lilongwe, Malawi; ${ }^{5}$ Bwaila District Hospital, STI Clinic, Lilongwe, Malawi; ${ }^{6}$ UNC Project Lilongwe, Laboratory, Lilongwe, Malawi; ${ }^{7}$ University of North Carolina at Chapel Hill, Division of Infectious Diseases, Chapel Hill, USA

\subsection{6/sextrans-2019-sti.846}

Background Malawi uses syndromic management for the treatment of sexually transmitted infections (STIs). However, the etiology profile of STIs has been shown to change over time. We conducted a current assessment for etiology of urethral discharge (UD) among men in Lilongwe Malawi to inform development of effective national treatment guidelines.

Methods We enrolled a cohort of HIV infected men with UD who were either ART naïve or on ART for $\geq 12$ weeks at Bwaila STI clinic. We collected blood samples and urethral swabs for STI etiologic testing as follows: Neisseria gonorrhoeae (GeneXpert, culture), Chlamydia trachomatis (GeneXpert), and Trichomonas vaginalis (OSOM - Trichomonas Rapid Test). All patients were treated syndromically with gentamicin, doxycycline, and metronidazole, per Malawian standard of care. Clinical and demographic characteristics were also collected. We assessed differences between men on ART for $\geq 12$ weeks and men not on ART using chi square tests and exact statistics (alpha $=0.05)$.

Results 189 men were enrolled between January 1, 2017 and December 31, 2018; 87 (46.0\%) were not on ART, and 102 (54.0\%) were on ART. Participants reported urethral discharge for a median of 4 days (IQR: 3, 7). Among participants, 152 $(80.4 \%)$ tested positive for gonorrhea via GeneXpert and 124 $(66.7 \%)$ via culture; 17 (9.0\%) tested positive for chlamydia, $6(3.2 \%)$ tested positive for trichomonas and $30(15.9 \%)$ did not test positive for any of the three etiologies. 15 (7.9\%) participants had multiple STIs. There were no differences in distribution of etiologies (individual or multiple) between men on and not on ART ( $\mathrm{p} \geq 0.10$ for all comparisons). 
Conclusion The overwhelming etiology of urethritis among HIV-infected men in Malawi is Neisseria gonorrhoeae. Current syndromic management guidelines that treat gonorrhea, chlamydia and trichomonas seem adequate for treatment of UD but future guidelines must be informed by ongoing monitoring of antibiotic resistance.

Disclosure No significant relationships.

\section{P793 RISK FACTORS FOR INCIDENT NON-GONOCOCCAL URETHRITIS (NGU) IN MEN WHO HAVE SEX WITH WOMEN (MSW) ATTENDING AN STD CLINIC}

\begin{abstract}
'Emily Rowlinson*, 'Laura Chambers, ${ }^{2}$ Sylvan Lowens, ${ }^{2}$ Jennifer Morgan, ${ }^{1}$ Tashina Robinson, 'Sarah Romano, 'Gina Leipertz, ${ }^{3}$ Matthew Golden, ${ }^{4}$ James Hughes, ${ }^{5}$ Lisa Manhart. 'University of Washington, Epidemiology, Seattle, USA; ${ }^{2}$ Public Health Seattle and King County, Seattle, USA; ${ }^{3}$ University of Washington, Medicine, Seattle, USA; ${ }^{4}$ University of Washington, Biostatistics, Seattle, USA; ${ }^{5}$ University of Washington, Epidemiology, Global Health, Seattle, USA
\end{abstract}

\subsection{6/sextrans-2019-sti.847}

Background Incidence and risk factors for NGU remain poorly defined. We conducted a cohort study to estimate incidence and identify associated risk factors in MSW.

Methods We enrolled cisgender male STD clinic patients age $\geq 16$, who reported exclusively female partners. At enrollment and six monthly follow-up visits, men underwent a clinical exam, provided urethral swab and urine specimens, and completed a sexual behavior survey. We tested for chlamydia (CT) and Mycoplasma genitalium (MG) using Aptima. NGU was defined as urethral symptoms or visible discharge plus $\geq 5$ polymorphonuclear leukocytes per high-power field on a Gram-stained slide. NGU following an NGU-negative visit was considered incident. We estimated incidence of NGU overall, pathogen-associated (MG or CT) and idiopathic NGU using Poisson regression for clustered outcomes. We performed relative risk binomial regression for clustered data to identify characteristics associated with incident NGU.

Results From 08/2014-08/2018, 254 participants had $\geq 1$ follow-up visit, contributing 100.6 person-years at risk during follow-up. Median age was 32 (range=17-71), 53\% were white and 24\% black. Eighty-four (33\%) had NGU at enrollment. Forty-five men had 53 cases of incident NGU (incidence $=0.53$ per person-year $(95 \%$ confidence interval $[\mathrm{CI}]$ $=0.39-0.71)$ ). Incidence of pathogen-associated and idiopathic NGU was 0.06 (95\% CI $0.03-0.13$ ) and 0.47 (95\%CI $=0.34-0.63)$, respectively. After adjustment for age, condom use and new partners during follow-up, risk of incident NGU was higher among black men (adjusted RR $(A R R)=2.2$; $95 \% \mathrm{CI}=1.1-4.4)$, those with a history of NGU before enrollment $(\mathrm{ARR}=3.1 ; 1.5-6.5)$ and more sex partners during follow-up $(A R R=1.2$ per partner; 1.0-1.5); risk was lower among men who used lubricant at last sex $(A R R=0.44$; 0.20-0.96).

Conclusion Incidence of NGU was high, predominantly idiopathic, and associated with traditional socio-behavioral characteristics, but not age, condom use, or new partners. The lubricant-use association was unexpected and warrants further exploration. More precise daily diary data may yield additional insights.

Disclosure No significant relationships.
P794 SIGNS AND SYMPTOMS ASSOCIATED WITH SINGLEPATHOGEN NONGONOCOCCAL URETHRITIS IN MEN

${ }^{1}$ Teresa Batteiger*, ${ }^{2}$ Stephen Jordan, ${ }^{3}$ Evelyn Toh, ${ }^{2}$ James Williams, ${ }^{3}$ Lora Fortenberry, ${ }^{1}$ Byron Batteiger, ${ }^{3}$ David Nelson. ${ }^{1}$ Indiana University School of Medicine, Medicine, Division of Infectious Diseases, Indianapolis, USA; ${ }^{2}$ Indiana University School of Medicine, Infectious Diseases, Indianapolis, USA; ${ }^{3}$ Indiana University School of Medicine, Microbiology and Immunology, Indianapolis, USA

\subsection{6/sextrans-2019-sti.848}

Background Syndromic management remains the standard nongonococcal urethritis (NGU) treatment approach. Whether pathogen-specific signs/symptoms inform treatment decisions remains unclear. We identified men with single- and mixedpathogen NGU and assessed for the presence of pathogen-specific signs or symptoms to improve syndromic management.

Methods As part of an ongoing cohort study (the Idiopathic Urethritis Men's Project [IUMP]), we recruited men with NGU. NGU was diagnosed by signs and/or symptoms of urethritis, and a urethral Gram stain with $\geq 5$ neutrophils per high-power field without evidence of gram negative intracellular diplococci. Participants underwent a clinical history and physical exam, which documented specific self-reported symptoms and clinician observed signs. Single- and mixed-infections were identified by NAAT testing of first-catch urine for Neisseria gonorrhoeae (NG), Chlamydia trachomatis (CT), Mycoplasma genitalium (MG), Trichomonas vaginalis (TV), and Ureaplasma urealyticum (UU); five-pathogen-negative cases were classified as idiopathic urethritis (IU).

Results One hundred fifty-five men with NGU are included in this analysis. The median age was 28 (range 18-63), 101 (65\%) were African American, and 135 (87\%) self-identified as heterosexual. The most commonly reported symptom was urethral discharge (92\%), followed by burning/tingling (37\%), and dysuria (28\%). Over half of these men reported more than one symptom (58\%). Single-pathogen NGU was detected in $99(64 \%)$ men, mixed-pathogen in $14(9 \%)$, and IU in 42 (27\%). For single pathogen NGU, 53 (34\%) had CT, 26 (17\%) had MG, 3 (2\%) had TV, and 17 (11\%) had UU. We compared single-pathogen NGU, mixed-infection and IU for differences in signs or symptoms and found no pathogen-specific differences.

Conclusion In men with NGU, no pathogen-specific signs and symptoms were identified that could inform treatment decisions. Pathogen-specific point-of-care tests are needed.

Disclosure No significant relationships.

\section{P795 PREVALENCE AND ETIOLOGY OF POST-AZITHROMYCIN PERSISTENT NON-GONOCOCCAL URETHRITIS (NGU) SYMPTOMS IN MEN}

${ }^{1}$ Stephen Jordan*, ${ }^{2}$ Evelyn Toh, ${ }^{3}$ Teresa Batteiger, ${ }^{1} J a m e s$ Williams, 2 Lora Fortenberry, ${ }^{1}$ Byron Batteiger, ${ }^{2}$ David Nelson. ${ }^{1}$ Indiana University School of Medicine, Medicine, Division of Infectious Diseases, Indianapolis, USA; 'Indiana University School of Medicine, Microbiology and Immunology, Indianapolis, USA; ${ }^{3}$ Indiana University School of Medicine, Indianapolis, USA

\subsection{6/sextrans-2019-sti.849}

Background Persistent NGU occurs when symptoms persist after empiric NGU treatment and has been associated with Mycoplasma genitalium (MG) infection. The prevalence and etiology of persistent NGU in men remains largely unknown. 